\title{
The Patient Experience of Fatigue in Systemic Lupus Erythematosus: A Conceptual Model
}

\author{
Sophie Cleanthous $\cdot$ Sara Strzok $\cdot$ Birgit Haier $\cdot$ Stefan Cano • \\ Thomas Morel
}

Received: July 7, 2021 / Accepted: September 6, 2021 / Published online: October 8, 2021

(c) The Author(s) 2021, corrected publication 2021

\begin{abstract}
Introduction: Fatigue is frequently experienced in systemic lupus erythematosus (SLE) and is a key outcome in clinical research trials. However, SLE fatigue is complex and poorly understood, and challenging to measure. We aimed to characterise fatigue from the patients' perspective and develop a conceptual model of fatigue based on qualitative interviews.

Methods: We conducted semi-structured qualitative interviews exploring fatigue in patients with SLE recruited from a social network $(n=29)$ and a phase 2 clinical study $(n=43)$. Transcripts were coded thematically, and codes were inductively categorised into a conceptual model.
\end{abstract}

Results: Fatigue was the most commonly reported symptom in the interviews and generated a wide range of codes. From these, our

Supplementary Information The online version contains supplementary material available at https:// doi.org/10.1007/s40744-021-00374-0.

S. Cleanthous $\cdot$ S. Strzok $\cdot$ S. Cano

Modus Outcomes, Letchworth Garden City, UK

B. Haier

UCB Pharma, Monheim, Germany

T. Morel $(\bowtie)$

UCB Pharma, Allée de la Recherche 60, 1070

Anderlecht, Brussels, Belgium

e-mail: Thomas.morel@ucb.com concept-driven approach revealed three overarching domains of the fatigue experienced in SLE: (i) physical manifestation of physical and bodily symptoms (including physical energy, stamina and impact on movement); (ii) mental and cognitive manifestation (including mental energy, motivation, and cognitive functioning symptoms); and (iii) susceptibility to fatigue or how easily 'fatigable' patients are, meaning how easily they become fatigued and how easily their fatigue is alleviated (including the rapid, disproportionate, and/or unpredictable onset of fatigue, non-restorative sleep, and need for more sleep/rest breaks). Within each of these, participants described the severity, variation and impact of fatigue on everyday life. Participants also described how the SLE fatigue experience differed from 'everyday tiredness'.

Conclusions: The findings of this research indicate that comprehensive measurement of fatigue in SLE will require consideration and quantification of the three domains described in our conceptual model. Future research will explore whether this conceptual model can form the basis of a valid and reliable measurement of fatigue in SLE.

Keywords: Autoimmune disease; Quality of life; Systemic lupus erythematosus 


\section{Key Summary Points}

\section{Why carry out this study?}

Fatigue is one of the most prevalent and debilitating symptoms in systemic lupus erythematosus (SLE), but remains a complex and poorly understood concept, posing both a treatment and measurement challenge.

Existing patient-reported outcomes used to measure fatigue in SLE may lack specificity or clarity, and this study is essential to further improve the assessment of fatigue in SLE.

This study aimed to characterise fatigue in SLE from the patients' perspective and to develop a conceptual model of fatigue in SLE with the potential to form the basis of a new patient-reported outcome instrument.

\section{What was learned from the study?}

This study revealed three overarching domains of the fatigue experience in SLE: physical, mental and cognitive and susceptibility to fatigue.

Based on these findings, a novel conceptual framework for capturing fatigue in SLE has been developed.

\section{INTRODUCTION}

Fatigue is one of the most prevalent and debilitating symptoms in systemic lupus erythematosus (SLE), described by patients as an overwhelming and unpredictable experience that can dominate their lives [1-6]. The report from the Lupus Patient-Focused Drug Development (PFDD) meeting held in 2017 flagged fatigue as the most burdensome SLE symptom, alongside joint and muscle pain and/or swelling [2]. 
performance of one of the most widely used fatigue PRO instruments in multiple sclerosis (MS), the Fatigue Impact Scale (FIS), an expert panel of 30 health professionals disagreed with the placement of $17 / 40$ of the items in the instrument's subscales. The health professionals considered all 40 items to be non-specific to fatigue, illustrating that while instruments can perform 'well' in terms of measurement, this is not sufficient if it is unclear what is actually being measured [17].

A recent systematic review evaluated widely used PRO instruments [20], including FACIT-F [18], Short-Form Health Survey (SF-36) [21, 22] and Lupus Quality of Life (LupusQoL) [23], that have been used to quantify fatigue in randomised controlled trials and observational studies in SLE. The review endorsed the use of these instruments in SLE, providing supportive evidence for their measurement properties, including reliability, convergent and face/content validity. However, this conclusion was based on secondary quantitative data. More recently a modern psychometric analysis [24] of these three widely used PRO instruments, specifically in relation to quantifying fatigue in SLE clinical trials, indicated some limitations such as the need to improve content validity of these instruments in assessing fatigue in SLE.

Specifically, the health-related quality of life instruments, SF-36 and LupusQoL, were found to be limited in the breadth of fatigue issues relevant in SLE. The FACIT-F [18] was found to lack conceptual clarity (i.e. inclusion of items related to 'impact of fatigue' vs fatigue itself) and there were test-design issues cited for fatigue-specific instruments, including the FACITF. Qualitative reviews of the FACIT-F instrument in SLE in specific samples confirmed the relevance of its content to the SLE fatigue experience [25], but also concluded that some items could be problematic in terms of conceptual relevance in SLE [26]. This further underlines the challenges of legacy PRO instruments in quantifying the specific concept of fatigue in SLE clinical trials.

To date, no study to the authors' knowledge has attempted to define SLE fatigue in a way that can reliably be used to select or develop a PRO instrument that is fit for purpose in SLE
$[7,8]$. We aimed to comprehensively conceptualise the experience of fatigue in SLE, using a bottom-up empirical approach, to understand fatigue from the perspective of the patients themselves.

\section{METHODS}

We carried out a two-phase qualitative investigation within a phenomenology framework, using thematic analysis to code interview transcripts. Phase 1 consisted of interviews with participants recruited through MyLupusTeam (www.myLupusTeam.com), a free social network for people in the USA diagnosed with SLE. Phase 2 consisted of interviews with US-based, English-speaking participants enrolled in an SLE phase 2 study (SL0023; NCT02804763). All sampling was purposive, with no specific cap on the number of interviews conducted.

\section{Compliance with Ethics Guidelines}

The SL0023 study protocol, amendments and patient informed consent were reviewed by a national, regional or independent ethics committee (IEC) or institutional review board (IRB). This study was conducted in accordance with the current version of the applicable regulatory and International Conference on Harmonisation-Good Clinical Practice requirements, the ethical principles that have their origin in the principles of the Declaration of Helsinki, and the local laws of the countries involved. Further details are provided below.

\section{Participants}

\section{Phase 1}

Participants were recruited online through MyLupusTeam, and eligibility to participate was confirmed using a self-report screener form. Eligible patients were at least 18 years of age, had a diagnosis of SLE and were currently receiving prednisone and hydroxychloroquine treatment. Ethical approval was granted by Copernicus Group Institutional Review Board (CGIRB) prior to any contact with participants, 
Table 1 Overview of sample characteristics

\begin{tabular}{lcl}
\hline & $\begin{array}{c}\text { Phase 1 } \\
(\boldsymbol{n}=\mathbf{2 9})\end{array}$ & $\begin{array}{l}\text { Phase 2/SL0023 } \\
(\boldsymbol{n}=\mathbf{4 3})\end{array}$ \\
\hline Age (years) & & \\
Range (mean, & $27-84(47.24$, & $25-70(44.23$, \\
SD) & $12.33)$ & $11.68)$ \\
Disease duration (years) & \\
Range (mean, & $0-46(9.90$, & $0.5-27.8(9.86$, \\
SD) & $10.07)$ & $8.32)$ \\
Gender, $n$ (\%) & $27(93.10)$ & $37(86.05)$ \\
Female & $2(6.90)$ & $6(13.95)$ \\
Male & $14(48.28)$ & $28(65.12)$ \\
Race/ethnicity, $n(\%)$ & $11(25.58)$ \\
White & $7(24.14)$ & $2(4.65)$ \\
Black/African & & $12(27.91)^{\mathrm{a}}$ \\
American & $7(24.14)$ & \\
Hispanic & $1(3.45)$ & \\
Mixed & & \\
Asian & & \\
\hline
\end{tabular}

${ }^{a}$ Hispanic or non-Hispanic ethnicity was captured as a separate variable within the SL0023 study

and informed consent was obtained before proceeding with the interview.

\section{Phase 2}

The phase 2 sample comprised patients enrolled in SL0023, a phase 2 clinical trial of dapirolizumab pegol (DZP) in moderately to severely active SLE [27]. Patients were randomised in the study to either placebo or DZP, in addition to standard of care therapies; both placebo- and DZP-randomised patients were included for interview, and interviewers were blinded to patient randomisation. Interviews were conducted during a \pm 3 -day window of their week 4 site visit. The interviews were included in the SL0023 clinical study protocol; ethical approval for these interviews was granted for all participating SL0023 study sites by the independent local IRB that reviewed the study and informed consent was obtained before proceeding with the interview.

\section{Interview Conduct}

All interviews were semi-structured telephone interviews (ca. $60 \mathrm{~min}$ ) conducted by an experienced qualitative interviewer. Interviews explored the experience of living with SLE, and specifically all relevant symptoms and impacts. Fatigue was a particular point of interest within the interviews (see examples of questions and probes in Supplementary Table S1). Interviews were recorded, anonymised and transcribed verbatim.

\section{Qualitative (Concept Elicitation) Analysis}

Transcripts were coded using ATLAS.ti software [28] and an open inductive coding approach, following general coding guidelines. Transcripts were coded by a team of two researchers $(\mathrm{SCl}$, SS) in phase 1 and by a team of four researchers (DE, NM, SCl, SS) in phase 2. To increase reliability, two transcripts in each phase were double coded, and codes were reviewed within the research teams. Researchers met regularly to discuss coding results and adjust coding guidelines as needed. Transcripts were analysed thematically [29] using detailed line-by-line open and inductive coding [30-32]. Following coding of all transcripts, standard analytical techniques of conceptual model development were used $[30,31,33]$. In an iterative process, codes and, where necessary, quotations were compared with the rest of the data and inductively categorised into higher-order overarching categories reflecting their conceptual content.

\section{RESULTS}

\section{Sample}

\section{Phase 1}

Of 32 enrolled participants, 29 were interviewed (May-June 2015; three participants cancelled their interview appointment). 27 (93\%) were female, with an age range of $27-84$ years, and 
time since diagnosis of $0-46$ years (Table 1 ). 17 of $29(59 \%)$ were taking immunosuppressant medications.

\section{Phase 2}

Of 46 US English-speaking participants enrolled in the SL0023 trial, 43 were interviewed (August 2016-January 2018; two did not reply and one exited the study prior to week 4). Thirty seven $(86 \%)$ were female, with an age range of 25-70 years (Table 1 ).

\section{Concept Elicitation}

Fatigue was the most commonly reported symptom, and generated a wide range of codes, highlighting its complexity and relevance in this population. Participants described aspects of the experience of fatigue, including (1) severity and severity variation; (2) variations between and within days including different triggers; (3) examples of how fatigue manifests, including the ways fatigue impacts daily activities and life. Many of these descriptions highlighted the patient-perceived difference between fatigue experienced whilst living with SLE and 'everyday tiredness'.

\section{SLE Fatigue Versus Ordinary Tiredness}

Some participants spontaneously used the term 'fatigue' to describe this key concept, while others used the term 'tired' or 'tiredness'. However, when specifically asked to describe the concept under consideration, participants made a clear distinction between the experience of ordinary or 'everyday' tiredness vs fatigue, or 'lupus tired'. Specifically, patients highlighted differences in severity, lack of direct cause, manifestation (both physical and mental) and increased severity during SLE flares that distinguished the experience of fatigue in SLE from the ordinary experience of tiredness (see below, and Table 2):

You have enough energy to still shower and go to bed [with ordinary tiredness], where this [SLE fatigue], is you don't care how filthy you are. You just go to bed. That's the difference

[Phase 1. Participant 03].

Fatigue is being tired and not knowing the reason for it. Being tired is from overexertion [Phase 1. Participant 11].

\section{SLE Fatigue Conceptual Model}

Our conceptual development approach revealed three overarching domains of the fatigue symptom experience. Two of these domains, physical fatigue and mental and cognitive fatigue, relate to distinct manifestations of fatigue. Physical fatigue was associated with the expression of fatigue in physical and bodily symptoms and sensations, while mental and cognitive fatigue was associated with the expression of fatigue in mental and cognitive symptoms and sensations. The third domain, susceptibility to fatigue, encompasses how 'fatigable' patients were in relation to both physical and mental/cognitive manifestations, i.e. how easily physical and mental/cognitive fatigue came about and how easily these manifestations were alleviated (Fig. 1). Within each of these domains, patients described experiences of fatigue that related to severity, variations, and impact of fatigue.

\section{Physical Fatigue}

When asked to define the experience of fatigue in SLE, many participants explained that a key component of fatigue was a lack of physical energy. Participants cited examples of activities that should require little energy to perform, but for which they needed to expend a disproportionate amount of energy or were unable to perform due to lack of energy:

Yeah, it means lack of energy to me. Sometimes you just feel exhausted [Phase 1. Participant 02].

...just the energy, how much energy it takes to just go, get in the shower, and wash up, because by the time I'm done showering, that's it. That's all the energy I had. Nothing else is left. I'm pretty much done for the day [Phase 2. Participant 626]. 
Table 2 Participant statements

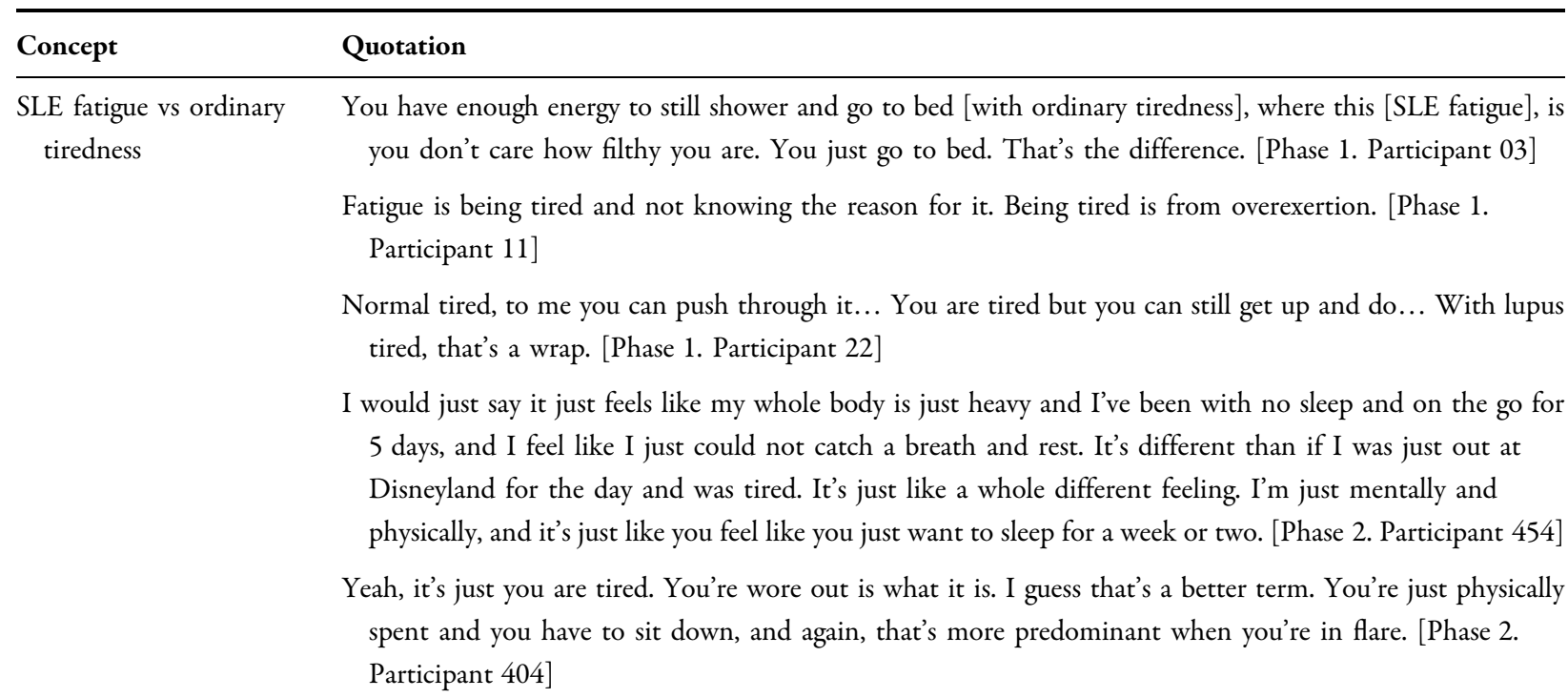

Physical fatigue

Yeah, it means lack of energy to me. Sometimes you just feel exhausted. [Phase 1. Participant 02]

Because I don't have a burst of energy to get up and do anything. I feel tired, and my muscles don't feel like they have a lot of strength in them. [Phase 1. Participant 20]

Just basically just the energy, how much energy it takes to just go, get in the shower, and wash up, because by the time I'm done showering, that's it. That's all the energy I had. Nothing else is left. I'm pretty much done for the day. [Phase 2. Participant 626]

Every day I'm very tired. It's like I barely have energy. I have to push myself to do everything, I have to push myself to get up and go to work. [Phase 2. Participant 517]

Fatigue is when I just can't get up and do nothing, like I've been-I know I got stuff to do and it's just my body would not let me get up. It's like somebody is literally holding me down, stopping me from getting up while I'm trying to fight to get up, and that is fatigue. [Phase 1. Participant 01]

You're really sluggish. It's hard to get up when your alarm goes off, and then you want to hit the snooze button as-you know, your body feels really heavy, like you're dragging it around, you know. You're making yourself walk when your body says no. [Phase 2. Participant 589]

Like I said, it's not good tiredness, like if you've ever worked out in the yard or whatever-end of the day, I've accomplished a lot. It's not like that. It really is like heaviness. It's kind of like having an anvil on your back and not being able to get it off. [Phase 1. Participant 25]

For one, and this is when it was at its most severe, is when I was initially diagnosed. I couldn't sit up. I couldn't stand up on my own. I had to literally roll onto the floor and crawl to the bathroom, or slither using my arms to pull my body. Because I couldn't stand, I couldn't pull myself up, and I had to have assistance just sitting up to sit on the toilet or sitting up to sit on the bed. So I basically had no control over my body when it comes to my limbs and the movement of my limbs. [Phase 1. Participant 24]

I just don't feel like, I'm tired. It's like I cannot lift my arm to grab my cup. [Phase 1. Participant 20]

You know, it's like the muscles just don't want to work, you know, the muscles in the brain, the muscles in my body. If I could find a fix for any of my symptoms, I would say the fatigue thing would be the thing I'd want. [Phase 2. Participant 485] 
Table 2 continued

\begin{tabular}{|c|c|}
\hline Concept & Quotation \\
\hline \multirow[t]{9}{*}{$\begin{array}{l}\text { Mental and cognitive } \\
\text { fatigue }\end{array}$} & $\begin{array}{l}\text { It's not just physical. To me, it's also a mental thing, because I think there's a mental energy that goes into } \\
\text { some things that you do, anyway. There are some things that don't require that, but most of what I do has } \\
\text { got some kind of mental aspect to it, as well. So, it's just being able to start and finish things. And I would } \\
\text { like to associate having energy with a positive feeling, as well. [Phase 1. Participant 05] }\end{array}$ \\
\hline & $\begin{array}{l}\text { I just don't have the energy to deal with that. And it's not necessarily-it's-I don't have the mental energy } \\
\text { to deal with it. Let me put it that way. I just don't have the motivation, if that's the right word. [Phase } 1 . \\
\text { Participant 21] }\end{array}$ \\
\hline & It just takes me longer to get motivated and do things because I'm so tired. [Phase 2. Participant 626] \\
\hline & $\begin{array}{l}\text { Not only was I exhausted but mentally I was exhausted. And I knew that I wasn't thinking clearly anymore. } \\
\text { [Phase 2. Participant 585] }\end{array}$ \\
\hline & $\begin{array}{l}\text { That's just more of a draggy kind of feeling. There's almost a brain fog, a fogginess that goes with the fatigue. } \\
\text { It makes it hard to put the sentences together, to concentrate, to function. [Phase } 1 \text {. Participant 05] }\end{array}$ \\
\hline & $\begin{array}{l}\text { And it's like I can be doing something and when this lupus stuff comes over, it's like a fog moves in and I } \\
\text { get-I don't know how to explain that, but it's just that I can't cope with what's going on around me. I } \\
\text { just want to go away, and I have trouble concentrating. Sometimes I don't even remember very vividly } \\
\text { what I've done. [Phase 1. Participant } 04 \text { ] }\end{array}$ \\
\hline & $\begin{array}{l}\text { It's almost like you're in a constant foggy daze, and you just can't focus, you can't think, you can't even read } \\
\text { something because you're looking at the words and you can't even make sense of the words. [Phase } 2 \text {, } \\
\text { Participant 626] }\end{array}$ \\
\hline & $\begin{array}{l}\text { Yes, definitely. Sometimes like in the afternoons when I'm more tired, it-start to just sort of lose focus, lose } \\
\text { concentration. They talk about that brain fog feeling, and that definitely also happens, usually in the } \\
\text { morning, though. I don't know quite how to describe it. [Phase 2. Participant 585] }\end{array}$ \\
\hline & $\begin{array}{l}\text { Typically, like I said, typically it's when my fatigue is extremely bad. And so, I would say that if it's one of } \\
\text { those days of, "I think I'm just going to go ahead and apologize before we even get started today, guys. } \\
\text { Write down your request for me." I don't really know if that correlates with the fatigue or not or if it's just } \\
\text { a cloudiness in my head that I just cannot focus. [Phase 2. Participant 437] }\end{array}$ \\
\hline \multirow[t]{2}{*}{ Susceptibility to fatigue } & $\begin{array}{l}\text { Yes. Like I said-and it's not all the time, but there are some days just very minimum activity wipes me out } \\
\text { completely. Just taking my son for a walk, for example, the other day, it put me on my back for the rest of } \\
\text { the night, like I just needed to lay down and relax after that, and then it became to the point that I could } \\
\text { barely keep my eyes open. [Phase } 1 \text {. Participant } 02 \text { ] }\end{array}$ \\
\hline & $\begin{array}{l}\text { Yeah, the fatigue is really bad because you can be fine in one minute, you can get up. Go to the restroom, } \\
\text { come back. It feels like you ran a mile. There are days where you can do a little more. You shower, you } \\
\text { dress yourself and you're okay. Within the next half hour, you could be doing nothing and it's just like } \\
\text { something hits you out of nowhere and you're so tired. [Phase 2. Participant 596] }\end{array}$ \\
\hline
\end{tabular}


Table 2 continued

\section{Concept Quotation}

... during the day just lack of energy, like doing things around the house. It's like I can do them at a slower pace and figuring out how to use my hands and whatnot, but then it's like I could easily take a nap after. Like if I go grocery shopping, two hours at the grocery store and coming home and putting it away, I'm exhausted. I'm like super tired and so I just want to go to sleep. [Phase 2. Participant 454]

You may have gotten up and slept the whole night, had a great night's sleep and wake up and say OK, I want to go back to bed now, and I don't want to do anything all day, and I want to be in my pyjamas and be lazy and not cook dinner and play with toys. So yeah, it has a lot of meanings depending on the day. [Phase 1. Participant 02]

It's being tired and not having a reason to be tired. You can't rest enough to feel better, and you're too tired to sleep, if that makes sense ... It's not so much you tire easily. You wake up tired [Phase 1. Participant 11]

I mean, you still feel exhausted all the time. Even if you sleep, you still wake up and feel like you needed more hours. [Phase 2. Participant 619]

Because if you're tired, you go to bed and you sleep, and you wake up and you feel refreshed. There's no sleeping and feeling refreshed when you're fatigued. [Phase 1. Participant 23]

Well, the only thing I can think of is I have what I call four levels of lupus issue. Number one issue is I'm tired every day, period. I don't care if I slept $8 \mathrm{~h}$ or $10 \mathrm{~h}$, I'm tired. So then if I decide, okay, well I don't feel too bad so I'm going to go accomplish a bunch of stuff for life today, then if I do too much, which is about three or four hours, then I go into what I call exhaustion which is just like okay, I need to stop and I need to not do anything else today. [Phase 2. Participant 695]

When I feel like really tired and I have to lay down during the day. Usually I have a lot of things going on in life. I have two kids, I have work, and if it turns to be that, I can't really do anything. I have to lay down on the couch all day, and I feel like my life is not as productive. [Phase 2. Participant 665]

Lupus fatigue is when you're tired, you don't want to do anything. When I wake up in the morning and sit up on my bed, I can't get up and just go. I have to wake-it's like waking your body up. You have to wake your body up. And so I sit there for a while, try to get my energy going to get out of bed, but then as soon as I get that moving around-say I want to go wash the dishes or straighten up a little bit, I have to do that about every two or three minutes. I just can't go fully and just go clean and then sit down. I have to take breaks maybe like every two or three minutes. [Phase 1. Participant 15]

Participants further described physical fatigue as a sense of lacking muscle strength, experiencing weakness in their limbs and a feeling of heaviness in their body. Some participants described how these physical manifestations limited movement. In some cases, patients reported that their bodies were difficult to move or even unresponsive due to the severity of their fatigue:

Fatigue is when I just can't get up and do nothing, like I've been-I know I got stuff to do and it's just my body would not let me get up. It's like somebody is literally holding me down, stopping me from getting up while I'm trying to fight to get up, and that is fatigue [Phase 1. Participant 01].

You're really sluggish. It's hard to get up when your alarm goes off, and then you want to hit the snooze button as-you know, your body feels really heavy, like you're dragging it around, you know. You're making yourself walk when your body says no [Phase 2. Participant 589].

\section{Mental and Cognitive Fatigue}

Participants made clear distinctions between the physical manifestations of fatigue and 


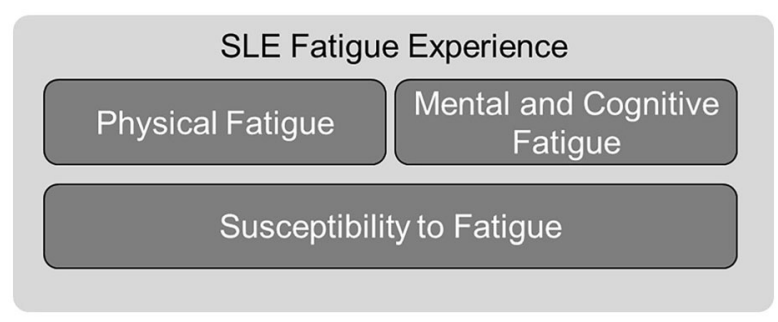

Fig. 1 Conceptual model of fatigue in SLE. Three overarching domains of the patient experience of fatigue were identified by the conceptual model: physical fatigue, mental and cognitive fatigue and susceptibility to fatigue. Susceptibility to fatigue related to how physically or mentally and cognitively 'fatigable' patients were

manifestations related to mental and cognitive fatigue. Specifically, participants described a sense of 'mental energy' (as opposed to general energy categorised in physical fatigue), which is often reduced when living with SLE. Participants often associated mental fatigue, mental exhaustion and lack of mental energy with a lack of or reduced motivation.

It's not just physical. To me, it's also a mental thing, because I think there's a mental energy that goes into some things that you do, anyway. There are some things that don't require that, but most of what I do has got some kind of mental aspect to it, as well. So, it's just being able to start and finish things. And I would like to associate having energy with a positive feeling, as well [Phase 1. Participant 05].

Participants depicted manifestations of mental and cognitive fatigue related to a state of 'brain fog' or fogginess where cognitive functioning is affected. Specifically, they described situations where fatigue manifests as difficulties in concentrating and focusing, thinking, remembering, and speaking, that they explicitly attribute to and link with fatigue.

That's just more of a draggy kind of feeling. There's almost a brain fog, a fogginess that goes with the fatigue. It makes it hard to put the sentences together, to concentrate, to function [Phase 1. Participant 05].

It's almost like you're in a constant foggy daze, and you just can't focus, you can't think, you can't even read something because you're looking at the words and you can't even make sense of the words [Phase 2. Participant 626].

Participants described the presence of both mental and cognitive fatigue, as well as physical fatigue, and some participants further indicated a different timeline of mental and cognitive fatigue both within and between days. For example, some participants indicated examples of mental and cognitive fatigue manifesting later in the day, or at times when they are generally more tired, in other words, linking mental and cognitive fatigue with a period of heightened overall fatigue.

...Sometimes like in the afternoons when I'm more tired, it-start to just sort of lose focus, lose concentration. They talk about that brain fog feeling, and that definitely also happens, usually in the morning, though. I don't know quite how to describe it [Phase 2. Participant 585].

\section{Susceptibility to Fatigue}

Whilst describing both physical and mental and cognitive symptoms of fatigue in SLE, participants described a further concept which related to both of these manifestations: susceptibility to fatigue. Susceptibility to fatigue refers to how 'fatigable' patients are, in relation to physical as well as mental and cognitive fatigue. First, patients described a lack of stamina, wherein they experience fatigue that is disproportionate to the degree of activity or effort expended. For example, patients described being exhausted by activities such as using the bathroom, showering, or taking a short walk. Such fatigue or lack of stamina can manifest suddenly, and is often unpredictable in its onset and across days:

Yes. Like I said-and it's not all the time, but there are some days just very minimum activity wipes me out completely. Just taking my son for a walk, for example, the other day, it put me on my back for the rest of the night, like I just needed to lay down and relax after that, and then it became to the point that I could barely keep my eyes open [Phase 1. Participant 02]. 
Yeah, the fatigue is really bad because you can be fine in one minute, you can get up. Go to the restroom, come back. It feels like you ran a mile [Phase 2. Participant 596].

Second, susceptibility to fatigue manifests in terms of non-restorative sleep and rest, where patients experience both physical as well as mental and cognitive fatigue even after having what objectively appears to be 'enough' sleep or rest. Many participants described situations that can be summarised as 'waking up feeling tired even after a full night's sleep', noting that achieving a long night's sleep does not necessarily translate into mental and physical energy with which to tackle the next day's tasks. In addition, participants described difficulty with waking up in the morning and mustering motivation to get out of bed, which is often a long process due to their fatigue:

It's being tired and not having a reason to be tired. You can't rest enough to feel better, and you're too tired to sleep, if that makes sense... It's not so much you tire easily. You wake up tired [Phase 1. Participant 11].

Many participants reported that it is issues related to fatigue susceptibility, including lack of stamina or energy reserves, the inability to replenish those reserves through sleep and rest, and the extent to which this fatigue susceptibility impacts their ability to accomplish daily tasks, that distinguish fatigue in SLE from 'everyday tiredness' as discussed above and further illustrated below:

When I feel like really tired and I have to lay down during the day. Usually I have a lot of things going on in life. I have two kids, I have work, and if it turns to be that, I can't really do anything. I have to lay down on the couch all day, and I feel like my life is not as productive [Phase 2. Participant 665].

\section{DISCUSSION}

Industry guidance from the FDA acknowledges that "improvements in clinical outcome measures (e.g. laboratory tests, clinical evaluation) in patients with SLE may not always translate to improvements in how patients feel or function" [14]. As such, the FDA states that any measurement of fatigue should rely on both a clear definition of fatigue, as it relates to patients with SLE, and a clear conceptual model describing the components of fatigue in SLE. Here, we propose such a framework.

We have conducted an extensive qualitative exploration of the experience of fatigue in patients with SLE. This work supports a new conceptual model of the fatigue phenomenon in SLE. Among participants from both the general SLE population and a clinical trial population, fatigue appeared as a key symptom of the SLE experience. Participants provided wideranging descriptions of fatigue, showcasing its complex and multi-dimensional nature. Participants characterised SLE fatigue as different from 'everyday tiredness' with distinctions drawn in terms of the severity levels of both physical fatigue and mental and cognitive fatigue, as well as other characteristics such as tiredness disproportionate to activity, sudden onset and unpredictability of fatigue, and nonrestorative rest and sleep.

The conceptual model derived from this research describes two manifestations of fatigue in SLE (i.e. physical fatigue, as well as mental and cognitive fatigue), as well as a general susceptibility to fatigue or how 'fatigable' patients are, in relation to both of these manifestations. Physical fatigue symptoms range from general lack of energy, to bodily signs including lack of strength, difficulty moving limbs and body, a sense of weakness, and heaviness. Mental and cognitive fatigue symptoms include a lack of mental energy and motivation, as well as a state of brain fog extending to difficulties with cognitive functioning. Whilst other studies have also suggested physical and mental/cognitive manifestations [1, 34, 35], this is the first study to report the concept of susceptibility to fatigue. According to patients, this susceptibility to fatigue is a key factor that differentiates SLE fatigue or 'lupus tired' from 'everyday tiredness' with distinctions drawn in terms of the severity levels of both physical fatigue and mental and cognitive fatigue. Susceptibility to fatigue, in other words how easily 'fatigable' patients feel in relation to both physical and mental/ 
cognitive manifestations, encompasses the ease with which patients get tired (more easily or earlier in the day), together with sudden or unpredictable onset of fatigue and the nonrestorative nature of sleep and rest, regardless of the amount of sleep or rest obtained. This echoes the patient testimonials presented during the externally led Lupus Patient-Focused Drug Development meeting - a parallel effort to the FDA's PFDD initiative, in which patients described SLE fatigue as not being able to ever 'fully recharge' [2]. One patient described the unpredictability and intensity of the fatigue experienced by many of the patients using the analogy of an old cell phone which no longer charges completely or reliably and can completely run down at any point in the day.

The conceptual model put forward by this study offers a detailed description of the phenomenon itself as opposed to its pattern, duration or frequency, impact, management, or triggers, which have often been the focus of previous work [1,36]. Additionally, participants provided insights regarding the relationship of the physical, and mental and cognitive manifestations. To our knowledge, this is the first study reporting how the two manifestations are related on the continuum of fatigue; this finding is in line with documented evidence of higher levels of fatigue being associated with depression and anxiety [37]. This finding provides new insights into the concept of fatigue and can support new hypotheses regarding the measurement of the concept in different contexts and populations. Most importantly, this study offers an in-depth conceptualisation of the fatigue experience in SLE that can offer the basis for fit-for-purpose measurement of fatigue, specific in the context of SLE. This diseasespecific conceptualisation addresses the unmet need in SLE fatigue measurement indicated by regulators [14] and could help to improve the measurement of this complex but important construct in SLE.

A strength of this study was the patientcentric methodology employed. Interviews included questions that focused on eliciting detailed descriptions of the experience of fatigue itself, as opposed to its impact, triggers, or other relevant aspects. Coding followed a detailed line-by-line approach, organically focused on the patients' own words and description of the fatigue experience, rather than pre-determined concepts or categories. Subsequently, codes were categorised inductively into higher-order domains, thus building a truly patient-focused conceptual model of fatigue $[30,31,33]$.

The study did have some limitations. Firstly, the recruitment for phase 1 was based on selfreporting and was not clinically defined. To mitigate this, self-reported treatment regimen information was collected as a proxy for disease severity. Secondly, all interviews were conducted in English. Therefore, further research is required to establish generalisability in other languages and cultures. Of note, parallel interviews with 52 additional Spanish-speaking participants were conducted within the SL0023 study in the USA and Latin America and these interviews were coded in Spanish. Results of these interviews were not reported here to avoid any potential inconsistencies and methodological challenges. However, results from these interviews were supportive of the model and fatigue hypothesis presented in this paper (further information is available upon request).

\section{CONCLUSIONS}

This study presents a conceptual model that describes the phenomenon of SLE fatigue and gathers patient data to illustrate how SLE fatigue differs from 'everyday tiredness', thus addressing gaps in previous research [16, 17]. These findings also indicate that comprehensive measurement of the symptom of fatigue in SLE will require consideration and quantification of dimensions described in our conceptual model. These include the physical manifestations of fatigue, mental and cognitive manifestations of fatigue and susceptibility to fatigue that encompasses rapid, disproportionate and/or unpredictable onset of fatigue, non-restorative sleep, and the need for more sleep or rest breaks. This research offers the first step towards the conceptualisation of this complex concept of interest. Future research will explore whether this conceptual model can form the basis of a 
valid and reliable measurement of fatigue in SLE.

\section{ACKNOWLEDGEMENTS}

The authors thank the patients who took part in this study.

Funding. This study was sponsored by UCB Pharma, Brussels, Belgium, and Biogen, Cambridge, MA, US. The rapid publication fee for Rheumatology and Therapy was funded by UCB Pharma, Brussels, Belgium, and Biogen, Cambridge, MA, US.

Medical Writing, Editorial, and Other Assistance. Support for third-party editorial assistance for this article, provided by Costello Medical Consulting, was funded by UCB Pharma, Brussels, Belgium, and Biogen, Cambridge, MA, US, in accordance with Good Publication Practice (GPP3) guidelines (http:// www.ismpp.org/gpp3). The authors acknowledge Patrick Marquis MD, MBA (Modus Outcomes, Cambridge, MA) for contributing to the initial study analysis and interpretation of results, Nadine McGale, PhD and Debi Ellis, MSc (both Modus Outcomes, Cambridge, MA) for data analysis. The authors also acknowledge Debbi Ellis, MSc and Nadine McGale, PhD for phase 2 interview transcription; Simone E Auteri, MSc EMS PhD (UCB Pharma, Brussels, Belgium) for publication coordination; and Helen Chambers, DPhil (Costello Medical, Cambridge, UK) for editorial assistance based on the authors' input and direction.

Authorship. All named authors meet the International Committee of Medical Journal Editors (ICMJE) criteria for authorship for this article and take responsibility for the integrity of the work as a whole. The authors had full editorial control of the manuscript and provided their final approval for all content in this version to be published. During the peer review process, Biogen and UCB Pharma had the opportunity to review the manuscript.
Author Contributions. Substantial contributions to study conception and design: $\mathrm{SCl}, \mathrm{SS}$, $\mathrm{BH}, \mathrm{SCa}, \mathrm{TM}$; substantial contributions to analysis and interpretation of the data: $\mathrm{SCl}, \mathrm{SS}$, $\mathrm{BH}, \mathrm{SCa}, \mathrm{TM}$; drafting the article or revising it critically for important intellectual content: $\mathrm{SCl}, \mathrm{SS}, \mathrm{BH}, \mathrm{SCa}, \mathrm{TM}$; final approval of the version of the article to be published: $\mathrm{SCl}, \mathrm{SS}, \mathrm{BH}$, SCa, TM.

Disclosures. Sophie Cleanthous, Sara Strzok and Stefan Cano are employees of Modus Outcomes, which received payment from UCB Pharma to conduct this research. Birgit Haier and Thomas Morel are employees and stockholders of UCB Pharma.

Compliance with Ethics Guidelines. The SL0023 study protocol, amendments, and patient informed consent were reviewed by a national, regional, or independent ethics committee or IRB. This study was conducted in accordance with the current version of the applicable regulatory and International Conference on Harmonisation (ICH)-Good Clinical Practice (GCP) requirements, the ethical principles that have their origin in the principles of the Declaration of Helsinki, and the local laws of the countries involved.

Data Availability. The datasets generated during and/or analysed during the current study are not publicly available.

Open Access. This article is licensed under a Creative Commons Attribution-NonCommercial 4.0 International License, which permits any non-commercial use, sharing, adaptation, distribution and reproduction in any medium or format, as long as you give appropriate credit to the original author(s) and the source, provide a link to the Creative Commons licence, and indicate if changes were made. The images or other third party material in this article are included in the article's Creative Commons licence, unless indicated otherwise in a credit line to the material. If material is not included in the article's Creative Commons licence and your intended use is not permitted by statutory regulation or exceeds the 
permitted use, you will need to obtain permission directly from the copyright holder. To view a copy of this licence, visit http:// creativecommons.org/licenses/by-nc/4.0/.

\section{REFERENCES}

1. Pettersson S, Moller S, Svenungsson E, Gunnarsson I, Welin HE. Women's experience of SLE-related fatigue: a focus group interview study. Rheumatology (Oxford). 2010;49:1935-42.

2. Lupus: Patient Voices. 2017. http://lupuspfdd.org/ LupusPatientVoicesFINAL.pdf. Accessed 14 Oct 2020.

3. Morgan C, Bland AR, Maker C, Dunnage J, Bruce IN. Individuals living with lupus: findings from the LUPUS UK Members Survey 2014. Lupus. 2018;27: 681-7.

4. Cleanthous S, Tyagi M, Isenberg D, Newman S. What do we know about self-reported fatigue in systemic lupus erythematosus? Lupus. 2012;21: 465-76.

5. Barbacki A, Petri M, Aviña-Zubieta A, Alarcón GS, Bernatsky S. Fatigue measurements in systemic lupus erythematosus. 2019. https://doi.org/10. 3899/jrheum.180831.

6. Azizoddin DR, Gandhi N, Weinberg S, Sengupta M, Nicassio PM, Jolly M. Fatigue in systemic lupus: the role of disease activity and its correlates. Lupus. 2019;28(2):163-73.

7. Food and Drug Administration. Guidance for Industry-Patient-Reported Outcome Measures: Use in Medical Product Development to Support Labeling Claims; 2009. https://www.fda.gov/ media/77832/download. Accessed 14 Oct 2020.

8. Food and Drug Administration. Roadmap to patient-focused outcome measurement clinical trials; 2013. https://www.fda.gov/media/87004/ download. Accessed 14 Oct 2020.

9. Strand V, Chu AD. Measuring outcomes in systemic lupus erythematosus clinical trials. Expert Rev Pharmacoecon Outcomes Res. 2011;11:455-68.

10. Izadi Z, Gandrup J, Katz PP, Yazdany J. Patient-reported outcome measures for use in clinical trials of SLE: a review. Lupus Sci Med. 2018;5:e000279.

11. Clowse ME, Wallace DJ, Furie RA, et al. Efficacy and safety of epratuzumab in moderately to severely active systemic lupus erythematosus: results from two phase III randomized, double-blind, placebocontrolled trials. Arthritis Rheumatol. 2017;69(2): 362-75.

12. Furie R, Petri MA, Strand V, Gladman DD, Zhong ZJ, Freimuth WW. Clinical, laboratory and health-related quality of life correlates of Systemic Lupus Erythematosus Responder Index response: a post hoc analysis of the phase 3 belimumab trials. Lupus Sci Med. 2014;1:e000031.

13. Khamashta M, Merrill JT, Werth VP, et al. Sifalimumab, an anti-interferon- $\alpha$ monoclonal antibody, in moderate to severe systemic lupus erythematosus: a randomised, double-blind, placebo-controlled study. 2016;75:1909-16.

14. Food and Drug Administration. Guidance for industry systemic lupus erythematosus; 2010. https://www.fda.gov/media/71150/download. Accessed 14 Oct 2020

15. Fisk JD, Pontefract A, Ritvo PG, Archibald CJ, Murray TJ. The impact of fatigue on patients with multiple sclerosis. Can J Neurol Sci. 1994;21:9-14.

16. Dittner A, Wesseley S, Brown R. The assessment of fatigue: a practical guide for clinicians. J Psychosom Res. 2004;56:157-70.

17. Hobart J, Cano S, Baron R, et al. Achieving valid patient-reported outcomes measurement: a lesson from fatigue in multiple sclerosis. Mult Scler J. 2013;19:1773-83.

18. Yellen SB, Cella DF, Webster K, Blendowski C, Kaplan E. Measuring fatigue and other anemia-related symptoms with the Functional Assessment of Cancer Therapy (FACT) measurement system. J Pain Symptom Manage. 1997;13:63-74.

19. Horisberger A, Courvoisier D, Ribi C. The Fatigue Assessment Scale as a simple and reliable tool in systemic lupus erythematosus: a cross-sectional study. Arthritis Res Ther. 2019;21(1):80.

20. Strand V, Simon LS, Meara AS, et al. Measurement properties of selected patient-reported outcome measures for use in randomised controlled trials in patients with systemic lupus erythematosus: a systematic review. Lupus Sci Med. 2020;7(1):e000373.

21. Ware JE, Kosinski M, Dewey JE. How to score version 2 of the SF-36 health survey. QualityMetric: Lincoln; 2000.

22. Ware JE Jr, Sherbourne CD. The MOS 36-Item Short-Form Health Survey (SF-36): I. Conceptual framework and item selection. Med Care. 1992;30: $473-83.25$ 
23. McElhone K, Abbott J, Shelmerdine J, et al. Development and validation of a disease-specific healthrelated quality of life measure, the LupusQol, for adults with systemic lupus erythematosus. Arthritis Rheum. 2007;57:972-9.

24. Cleanthous S, Regnault A, Schneider M, et al. Measuring what matters to lupus patients: translating patient views into novel patient-reported outcomes. Ann Rheum Dis. 2018;77:A1464.

25. Raymond K, Park J, Joshi A, et al. Patient experience with fatigue and qualitative interview-based evidence of content validation of The FACIT-fatigue in systemic lupus erythematosus. Rheumatol Ther. 2021;8(1):541-54.

26. Kosinski M, Gajria K, Fernandes AW, et al. Qualitative validation of the FACIT-fatigue scale in systemic lupus erythematosus. Lupus. 2013;22(5): 422-30.

27. Furie R, Bruce IN, Dorner T, et al. Efficacy and safety of dapirolizumab pegol (DZP) in patients with moderately to severely active systemic lupus erythematosus (SLE): a randomised, placebo (PBO)controlled study. Ann Rheum Dis. 2019;78:A775.

28. Friese S. ATLAS.ti 7 User Guide and Reference; 2013.

29. Braun V, Clarke V. Using thematic analysis in psychology. Qual Res Psychol. 2006;3:77-90.

30. Bowling A. Research methods in health: investigating health and health services. 3rd ed. Maidenhead: Open University Press; 2009.
31. Bryman A, Burgess B. Analyzing qualitative data. New York: Routledge; 2002.

32. Thomas DR. A general inductive approach for analyzing qualitative evaluation data. Am J Eval. 2006;27:237-46.

33. Klassen AF, Pusic AL, Scott A, Klok J, Cano SJ. Satisfaction and quality of life in women who undergo breast surgery: a qualitative study. BMC Women's Health. 2009;9:11-8.

34. Beckerman NL. Living with lupus: a qualitative report. Soc Work Health Care. 2011;50:330-43.

35. Ow YL, Thumboo J, Cella D, Cheung YB, Yong Fong $\mathrm{K}$, Wee HL. Domains of health-related quality of life important and relevant to multiethnic Englishspeaking Asian systemic lupus erythematosus patients: a focus group study. Arthritis Care Res (Hoboken). 2011;63:899-908.

36. Sterling K, Gallop K, Swinburn P, et al. Patient-reported fatigue and its impact on patients with systemic lupus erythematosus. Lupus. 2014;23: 124-32.

37. Arnaud L, Gavand PE, Voll R, et al. Predictors of fatigue and severe fatigue in a large international cohort of patients with systemic lupus erythematosus and a systematic review of the literature. Rheumatology. 2018;58:987-96. 